\title{
Conditions for Isolation of and Colony Formation by Mycelial Protoplasts of Coprinus macrorhizus
}

\author{
Sonoe Ochiai Yanagi, Mitsuru Monma, Toshiyuki Kawasumi, Akihiro Hino, \\ Masako Kito and Itaru TAKEBE* \\ National Food Research Institute, Ministry of Agriculture, Forestry and Fisheries, \\ Yatabe-machi, Tsukuba-gun, Ibaraki 305, Japan \\ *Faculty of Science, Nagoya University, \\ Furo-cho, Chikusa-ku, Nagoya 464, Japan
}

Received August 13, 1984

\begin{abstract}
Conditions suitable for the release of mycelial protoplasts of Coprinus macrorhizus and for colony formation by the isolated protoplasts were examined. The study culminated in the development of a method using commercially available preparations of chitinase, cellulase and $\beta$ glucanase, by which over $2 \times 10^{8} / \mathrm{ml}$ protoplasts could be obtained within $2 \mathrm{hr}$. The protoplasts from the wild type and mutant $\mathrm{Fis}^{\mathrm{c}}$ of $C$. macrorhizus regenerated into hyphae in a complex medium containing malt and yeast extract, and subsequently formed colonies with frequencies up to $50 \%$, which paralleled the percentage of nucleated protoplasts. The plating efficiency in a minimal medium was about $20 \%$ on average. Sucrose was found to be superior to mannitol as an osmoticum for colony formation. Protoplasts from auxotrophic mutants formed colonies at much lower frequencies even in the complex medium, but addition of $\mathrm{N}$-acetyl glucosamine significantly improved their plating efficiency. Colonies derived from the protoplasts of C. macrorhizus Fis $^{\mathrm{c}}$ consistently developed fruiting bodies, when grown on a hypotonic medium.
\end{abstract}

Protoplasts are widely used in the fields of basic and applied genetics of microorganisms such as bacteria, yeasts and filamentous fungi, because the wall-less form of cells permits gene transfer via such unconventional means as cell fusion and transformation. ${ }^{1,2)}$ The potential of protoplasts has not so far been explored in basidiomycetes, a group of microorganisms important in the study of morphogenesis and as foodstuffs. This seems to be due partly to the absence of an established method for isolating sufficiently large numbers of basidiomycete protoplasts with a high developmental potential.

There have been several reports on the isolation of basidiomycete protoplasts. Wessels and coworkers studied the composition of hyphal walls of Schizophyllum commune, and showed that protoplasts can be isolated by digesting the mycelia with the culture filtrate of Trichoderma viride grown on the hyphal walls of $S$. commune, ${ }^{3,4)}$ or with $\alpha$-glucanase and chitinase purified from the culture filtrate. ${ }^{5)}$ Their method involved, however, preparation of lytic enzymes, required prolonged digestion, and yielded relatively small numbers of protoplasts $\left(10^{6} / \mathrm{ml}\right)$. Protoplasts have since then been isolated from Coprinus cinereus, ${ }^{*, 7,8)}$ Lentinus edodes, ${ }^{9}$ Tricholoma matsutake, ${ }^{10)}$ Collybia veltipes, ${ }^{11)}$ and Pleurotus ostreatus, ${ }^{11)}$ but they are poor either in yield or in their capacity to regenerate into hyphae. Furthermore, there has been no report of development of isolated basidiomycete protoplasts up to the stage of fruiting bodies.

In a recent paper we briefly reported an efficient method for isolating mycelial protoplasts of Coprinus macrorhizus and several other basidiomycete species. ${ }^{12)}$ The present

Abbreviations: MES, 2-( $N$-morpholino)-ethane sulfonic acid; DAPI, 4,6-diamidino-2-phenylindole.

* According to a report by Moore et al., ${ }^{6}$ C. cinereus is the same species as C. macrorhizus. 
paper contains a full account of the method with some modifications, documents conditions favoring colony formation by the isolated $C$. macrorhizus protoplasts, and reports development into fruiting bodies of protoplasts from a mutant strain.

\section{MATERIALS AND METHODS}

Organisms. Coprinus macrorhizus Fis $^{\mathrm{c}}$, a mutant capable of forming monokaryotic fruiting bodies, ${ }^{13)}$ was obtained from Dr. T. Ishikawa, Institute of Applied Microbiology, University of Tokyo, and was used in most of the experiments. Auxotrophic mutants, C. macrorhizus 5010 (his $^{-}$) and $5132\left(\mathrm{ade}^{-}\right)$were kindly provided by Dr. Takemaru, Faculty of Science, Okayama University, and wild type $C$. macrorhizus was obtained from Mr. M. Saito, National Food Research Institute, Ministry of Agriculture, Forestry and Fisheries. The organisms were maintained on $1.5 \%$ agar slants of a medium containing per liter $10 \mathrm{~g}$ malt extract (Difco), $4 \mathrm{~g}$ yeast extract (Difco) and $4 \mathrm{~g}$ glucose (MYG medium).

Isolation of protoplasts. Spores, oidia and small fragments of hyphe, obtained by washing the surface of a slant culture, were passed through a nylon bolting cloth (mesh opening, $120 \mu$ ) and inoculated into $20 \mathrm{ml}$ of MYG medium in $100 \mathrm{ml}$ conical flasks. Cultures were performed at $30^{\circ} \mathrm{C}$ without shaking and under illumination with white fluorescent lamps (ca. 2,000lux) with a $12 \mathrm{hr}$ light and $12 \mathrm{hr}$ dark cycle. Mycelia grown after $50 \sim 80 \mathrm{hr}$ culture were harvested and pressed gently with a spatula to remove the medium. Mycelia of $100 \sim 400 \mathrm{mg}$ wet weight were transferred to $1 \mathrm{ml}$ of enzyme solution in a short test tube, which was vortexed for $2 \sim 3 \mathrm{sec}$. Incubation was performed in a water bath of $30^{\circ} \mathrm{C}$ with reciprocal shaking (stroke $3 \mathrm{~cm}$ ) at a frequency of 80 revolutions $/ \mathrm{min}$. At $30 \mathrm{~min}$ intervals, the reaction mixture was vortexed for $2 \sim 3 \mathrm{sec}$, a sample was withdrawn, and the number of released protoplasts was determined in a haemacytometer. The standard enzyme solution contained $0.1 \sim 0.2 \%$ (4 units $/ \mathrm{ml}$ ) of chitinase (Sigma), $2 \%$ Cellulase Onozuka $\mathbf{R}$ $10,0.1 \%$ Zymolyase $60,000,0.5 \mathrm{M}$ mannitol and $50 \mathrm{~mm}$ maleate buffer, $\mathrm{pH}$ 5.5. After the enzymes were dissolved, the $\mathrm{pH}$ was readjusted to 5.5 , and insoluble matter was removed by centrifugation at $1,500 \times g$ for $10 \mathrm{~min}$. The enzyme solution was filter-sterilized using a membrane filter (pore size, $0.22 \mu \mathrm{m}$ ) when protoplasts were to be isolated aseptically.

Cytological methods. Calcofluor White ST, a fluorescent dye with affinity to cellulose and chitin, ${ }^{14)}$ was used to monitor digestion of mycelial walls. Samples were stained with $0.1 \%$ Calcofluor White ST in $0.5 \mathrm{M}$ mannitol solution according to the procedure of Nagata and Takebe, ${ }^{15)}$ and were examined under an epi-illumination type fluorescence microscope (Olympus Vanox AH-RFL-LB) using a UG-1 exciter filter and a 650 barrier filter. Nuclei were stained with 4,6-diamidino-2-phenylindole (DAPI) at a concentration of $1 \mu \mathrm{g} / \mathrm{ml}$ essentially according to the method of Kuroiwa and Suzuki. ${ }^{16)}$ Protoplasts in a thick suspension were fixed with ten volumes of $72 \%$ ethanol solution containing $12 \%$ acetic acid and $0.2 \mathrm{~m}$ sorbitol. The fixative solution was added dropwise and very gently over a $30 \mathrm{~min}$ period.

Culture of protoplasts. Protoplasts aseptically isolated by $2 \mathrm{hr}$ digestion with enzyme solutions were passed through a sheet of nylon bolting cloth (mesh opening, $60 \mu$ ), and serially diluted to $10^{-5}$ with regeneration medium. The standard regeneration medium was MYG containing $0.5 \mathrm{M}$ sucrose. Two hundred $\mu \mathrm{l}$ of the diluted suspension of protoplasts was spread on $1 \%$ agar plates of the same medium in $6 \mathrm{~cm}$ Petri dishes. For liquid cultures, $1 \sim 3 \mathrm{ml}$ of a protoplast suspension of a desired concentration was poured into Petri dishes. Incubation was performed as described above for the growth of mycelia. Plating efficiency was expressed as the percentage of protoplasts that formed colonies after 4 days culture. The minimal medium contained per liter $20 \mathrm{~g}$ glucose, $0.5 \mathrm{~g}$

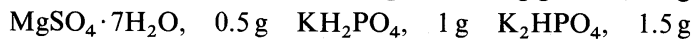
$\left(\mathrm{NH}_{4}\right)_{2} \mathrm{HPO}_{4}$ and $120 \mu \mathrm{g}$ thiamine-- $\mathrm{HCl}^{8}{ }^{8}$

Enzymes. The following enzymes were used; chitinase from Streptomyces griseus (Sigma Chemical Co., St. Louis, Missouri, U.S.A.) chitinase from Streptomyces antibioticus (Calbiochem-Behring, San Diego, California U.S.A.) chitinase from Streptomyces sp. (ICN Nutritional Biochem., Cleveland, Ohio, U.S.A.), Cellulase Onozuka R 10 and RS from Trichoderma viride (Yakult Honsha, Nishinomiya, Japan), $\beta$-glucuronidase from Helix pomatia (Sigma Chemical Co.), Helicase (Pharmindustrie, France), Zymolyase 60,000 from Arthrobacter luteus (Seikagaku Kogyo, Nihonbashi, Tokyo, Japan), Macerozyme R 10 from Rhizopus sp. (Yakult Honsha) and Novozyme 234 from Trichoderma harzianum (Novo Industry, Japan, Chiyoda-ku, Tokyo).

\section{RESULTS}

\section{Factors influencing release of protoplasts}

Source tissue. Corinus macrorhizus $\mathrm{Fis}^{\mathrm{c}}$ was used in all the experiments unless otherwise mentioned. Mycelia of this organism grew in the static culture initially in the liquid phase; the growth of aerial hyphae became significant only after $70 \mathrm{hr}$ culture. Since the aerial hyphae were relatively resistant to digestion by cell wall lytic enzymes, mycelial mats harvested between 50 and $80 \mathrm{hr}$ culture were used as the source of protoplasts. Mycelial pellets formed 
by shake cultures were resistant to digestion without prior homogenization, which reduced the viability of mycelial cells markedly.

Osmoticum: The effects of osmotic conditions on the release of protoplasts were studied using a mixture of Cellulase Onozuka R 10 and Sigma chitinase (Combination $C$ in Table I). Sorbitol, mannitol, pentaerythritol, $\mathrm{MgSO}_{4}$ and $\mathrm{KCl}$ at $0.5 \mathrm{M}$ were all effective as osmotic stabilizers and gave similar yields of protoplasts. These results differ from those of DeVries and Wessels ${ }^{3)}$ who reported that $\mathrm{MgSO}_{4}$ and $\mathrm{KCl}$ give much higher yields of $S$. commune protoplasts than sorbitol and mannitol. Mannitol was chosen as the osmotic stabilizer, because protoplasts of C. macrorhizus appeared to be most stable with this osmoticum when maintained overnight. With mannitol as the osmoticum, protoplasts were obtained in the highest yields at $0.5 \mathrm{M}$ during the first $2 \mathrm{hr}$ of enzyme treatment. When digestion lasted for several hours, however, somewhat higher yields resulted, $0.6 \sim 0.7 \mathrm{M}$ than with $0.5 \mathrm{M}$ mannitol.

Later during the course of this study sucrose was also tested as an osmoticum. The yield of protoplasts was somewhat lower in $0.5 \mathrm{M}$ sucrose than in $0.5 \mathrm{M}$ mannitol, but the protoplasts isolated using sucrose showed higher frequencies of colony formation.

Buffer and $p H$. Maleate, succinate and MES could be used as buffers for the enzyme solution. In contrast, acetate buffer gave poor yields of protoplasts. With maleate buffer, the yield of protoplasts was highest at $\mathrm{pH} 5.5$ throughout the enzyme reaction.

Table I. Release of Protoplasts from Coprinus macrorhizus Mycelia Using Various Enzyme Preparations Singly and in Combination ${ }^{a}$

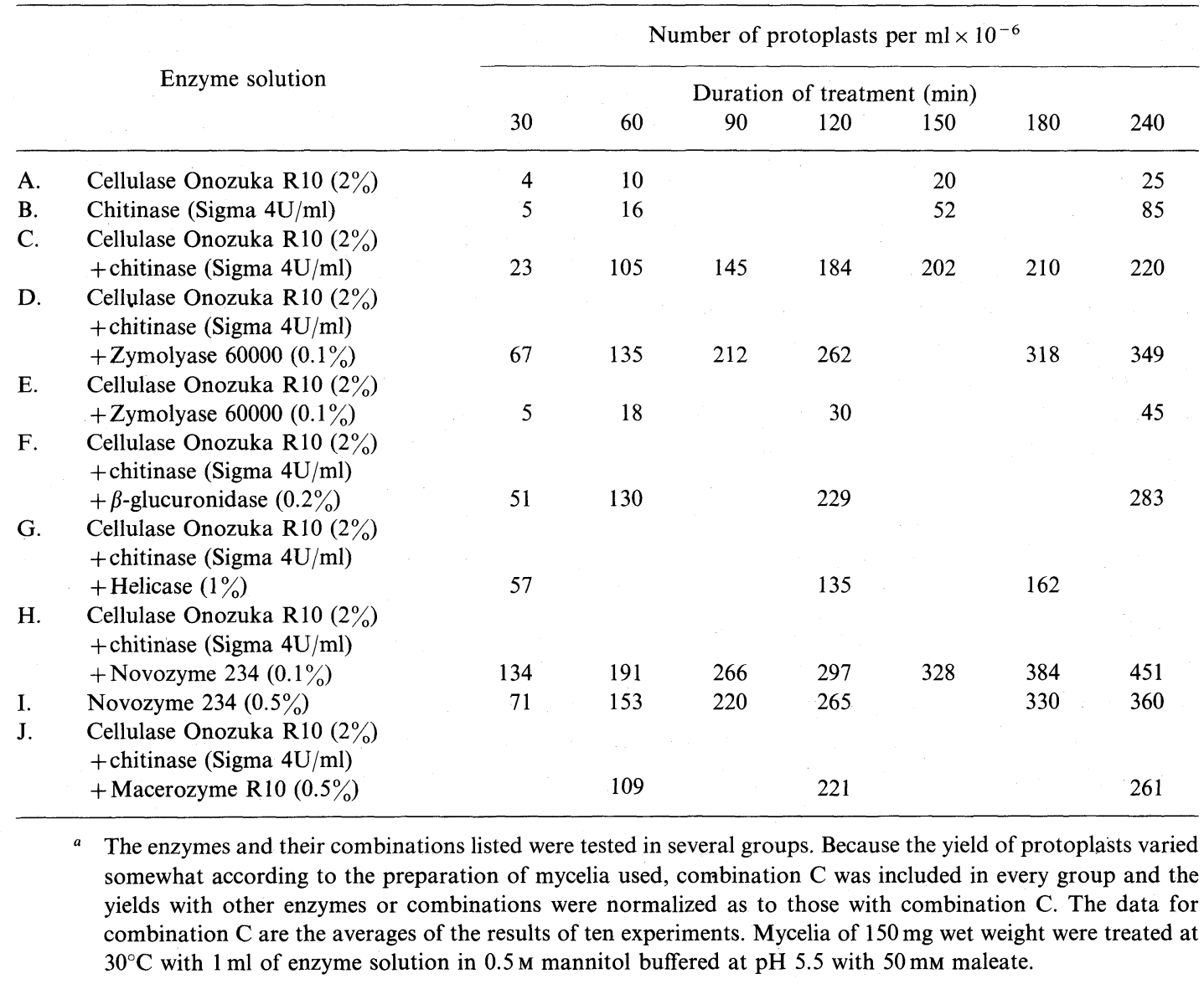


Table II. Percentage Nucleation and Plating Efficiency of Coprinus macrorhizus Protoplasts Isolated Using Various Combinations of EnZymes

\begin{tabular}{|c|c|c|c|c|c|c|}
\hline \multirow{2}{*}{$\begin{array}{l}\text { Duration of } \\
\text { treatment } \\
\text { (min) }\end{array}$} & \multicolumn{2}{|c|}{$\mathrm{CC}^{a}$} & \multicolumn{2}{|c|}{$\mathrm{CCZ}^{b}$} & \multicolumn{2}{|c|}{$\mathrm{CCN}^{c}$} \\
\hline & $\begin{array}{c}\text { Nucleated } \\
\text { protoplasts } \\
(\%)\end{array}$ & $\begin{array}{l}\text { Plating }^{d} \\
\text { efficiency } \\
(\%)\end{array}$ & $\begin{array}{c}\text { Nucleated } \\
\text { protoplasts } \\
(\%)\end{array}$ & $\begin{array}{l}\text { Plating }^{d} \\
\text { efficiency } \\
(\%)\end{array}$ & $\begin{array}{c}\text { Nucleated } \\
\text { protoplasts } \\
(\%)\end{array}$ & $\begin{array}{c}\text { Plating }^{d} \\
\text { efficiency } \\
(\%)\end{array}$ \\
\hline 30 & 41 & 26 & 39 & 27 & 32 & 18 \\
\hline 60 & 41 & 32 & 38 & 34 & 30 & 28 \\
\hline 120 & 47 & 37 & 49 & 39 & 36 & 31 \\
\hline 240 & 48 & 37 & 50 & 38 & 37 & 31 \\
\hline
\end{tabular}

a Cellulase Onozuka R10 $(2 \%)+$ chitinase (Sigma $4 \mathrm{U} / \mathrm{ml})$.

$b$ Cellulase Onozuka R10 $(2 \%)+$ chitinase (Sigma 4U/ml) + Zymolyase $60000(0.1 \%)$.

c Cellulase Onozuka R10 $(2 \%)+$ chitinase (Sigma 4U/ml) + Novozyme $234(0.1 \%)$.

$d$ Protoplasts were plated on agar plates of MYG medium containing $0.5 \mathrm{M}$ mannitol.

Enzymes. Various commercial enzyme preparations were tested as to their ability to release mycelial protoplasts when used singly and in combinations, and the results are shown in Table I. Cellulase Onozuka R 10 alone released few protoplasts (A), whereas Sigma chitinase yields significant numbers of protoplasts after $4 \mathrm{hr}$ treatment (B). The yield of protoplasts was strikingly increased when these enzymes were used in combination (C); as many as $10^{8}$ protoplasts were released per $\mathrm{ml}$ within $1 \mathrm{hr}$. Chitinase from Calbiochem Behring was as effective as Sigma chitinase when used in combination with Cellulase Onozuka, whereas ICN chitinase was much less effective (data not shown). Cellulase Onozuka RS was no better than R 10 . Increasing the concentration of Cellulase Onozuka R 10 or chitinase over those specified did not improve the yield of protoplasts significantly, and lower concentrations were less effective.

When the mixture of Cellulase Onozuka $R$ 10 and Sigma chitinase was further supplemented with Zymolyase 60,000 (D) or with Novozyme $234(\mathrm{H})$, the yield of protoplasts increased significantly. A similar but less pronounced effect was seen with $\beta$-glucuronidase (F) and Macerozyme R 10 (J). Unexpectedly, Helicase added to the mixture reduced the yield of protoplasts (G). Novozyme 234 released large numbers of protoplasts even when used singly (I). The protoplasts released using Novozyme (H, I) were, however, relatively small in size and there was a larger proportion of enucleated protoplasts (Table II). Addition of dithiothreitol, known to enhance the lytic activity of Zymolyase toward yeasts, adversely affected the release of Coprinus protoplasts (data not shown).

Based on the results described above, the standard enzyme solution used for the isolation of $C$. macrorhizus protoplasts was as follows: $2 \%$ Cellulase Onozuka R 10, 4 U/ml $(0.1 \sim 0.2 \%)$ Sigma chitinase, $0.1 \%$ Zymolyase $60,000,50 \mathrm{~mm}$ maleate buffer $(\mathrm{pH} 5.5), 0.5 \mathrm{M}$ mannitol.

The enzyme reaction was carried out usually at $30^{\circ} \mathrm{C}$. At $32^{\circ} \mathrm{C}$ and $28^{\circ} \mathrm{C}$, a slight increase and a significant decrease in protoplast yield were observed, respectively.

It should be added that the standard enzyme solution was effective also for other mushroom species, although the yields of protoplasts were somewhat lower as compared with C. macrorhizus. The species tested included Flammulina velutipes, Ganoderma lucidum, Grifola frondosa, Lentinus edodes, Lyophyllum ulmarium, Pleurotus ostreatus, Pholiota nameko and Tremella fuciformis. The yield of protoplasts ranged from 0.5 to $10 \times 10^{7} / \mathrm{ml}$.

\section{Cytological observations}

When $C$. Macrorhizus mycelia were treated 

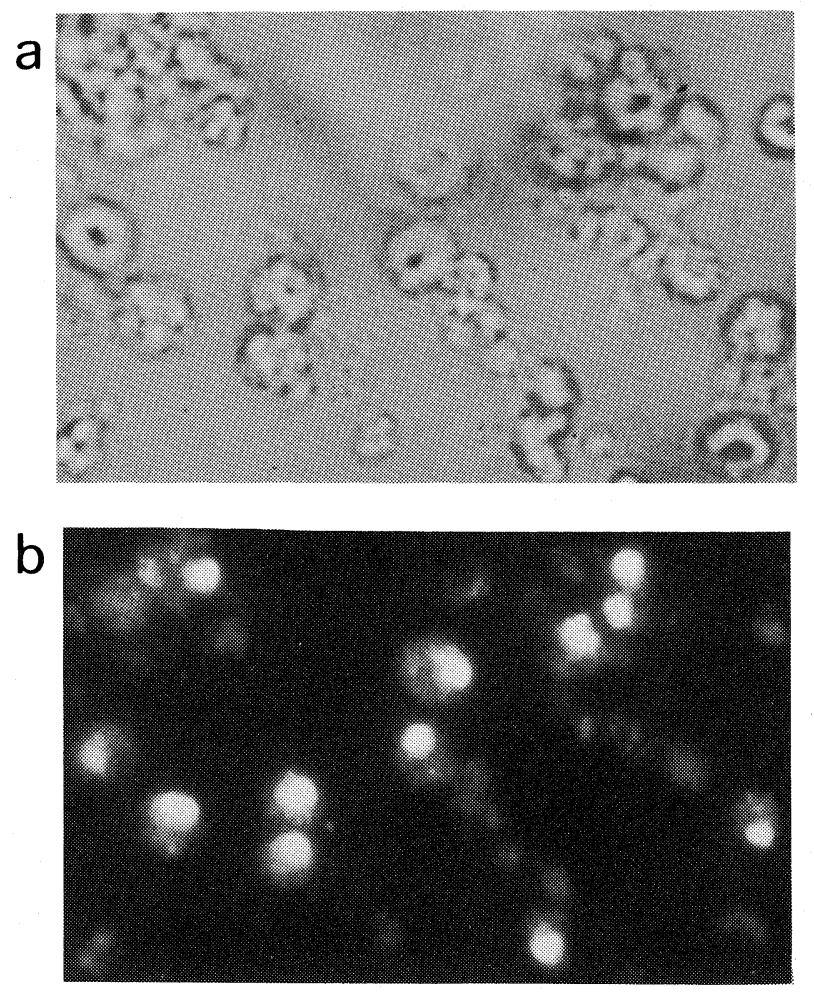

FIG. 1. Mycelial Protoplats of Coprinus macrorhizus Fis $^{\mathrm{c}}$ Stained with DAPI.

a: Ordinary light micrograph.

b: Fluorescence micrograph of the same field as in Fig. 1a.

with the standard enzyme solution, protoplasts were initially released from hyphal tips. The enzymes later solubilized a substantial portion of the entire mycelia releasing numerous protoplasts. The absence of walls in the isolated protoplasts was indicated by the observations that they burst in hypotonic solutions and that they were not stained with Calcoflour White. Walls of mycelia fluoresced brightly blue upon staining with this dye. Protoplasts varied considerably in size $(2 \sim 10 \mu)$ (Fig. 2a). The heterogeneity in size presumably reflects the varying ages of the mycelial cells from which protoplasts were released, and may also result from pinching off of cytoplasm during the release of protoplasts. ${ }^{3)}$ Staining with DAPI showed the presence of nuclei in approximately $50 \%$ of protoplasts harvested after $2 \mathrm{hr}$ incubation (Table II) (Fig. 1).

\section{Development of isolated protoplasts}

Protoplasts isolated from the mycelia of $C$. macrorhizus regenerated into hyphae when cultured as a suspension in liquid MYG medium, or spread on or embedded in agar MYG plates containing osmotic stabilizers. Apparently normal hyphae grew directly from the protoplasts (Fig. 2b, c); growth of hyphae from bud-like structures ${ }^{4}$ or in abnormal forms was observed only rarely and mostly in suspension cultures (Fig. 3g i). The hyphae originated from the protoplasts developed into colonies within 3 or 4 days (Fig. 3d). Few, if any, colonies were formed when protoplasts were plated on the same medium containing no osmoticum. The small number of colonies (less than $1 \%$ of the plated protoplasts) occasionally formed on such plates should be due to small fragments of residual mycelia which passed through the nylon mesh and thus con- 


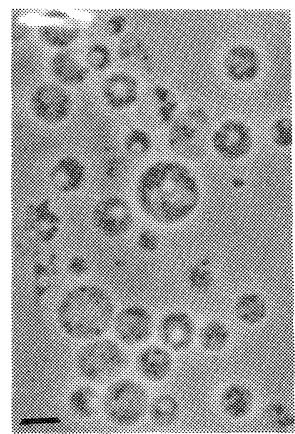

a

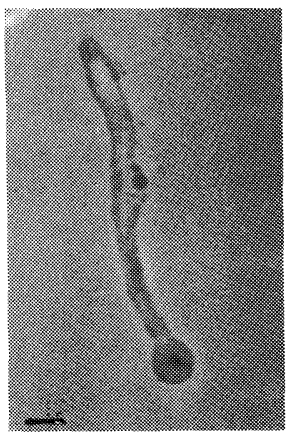

b
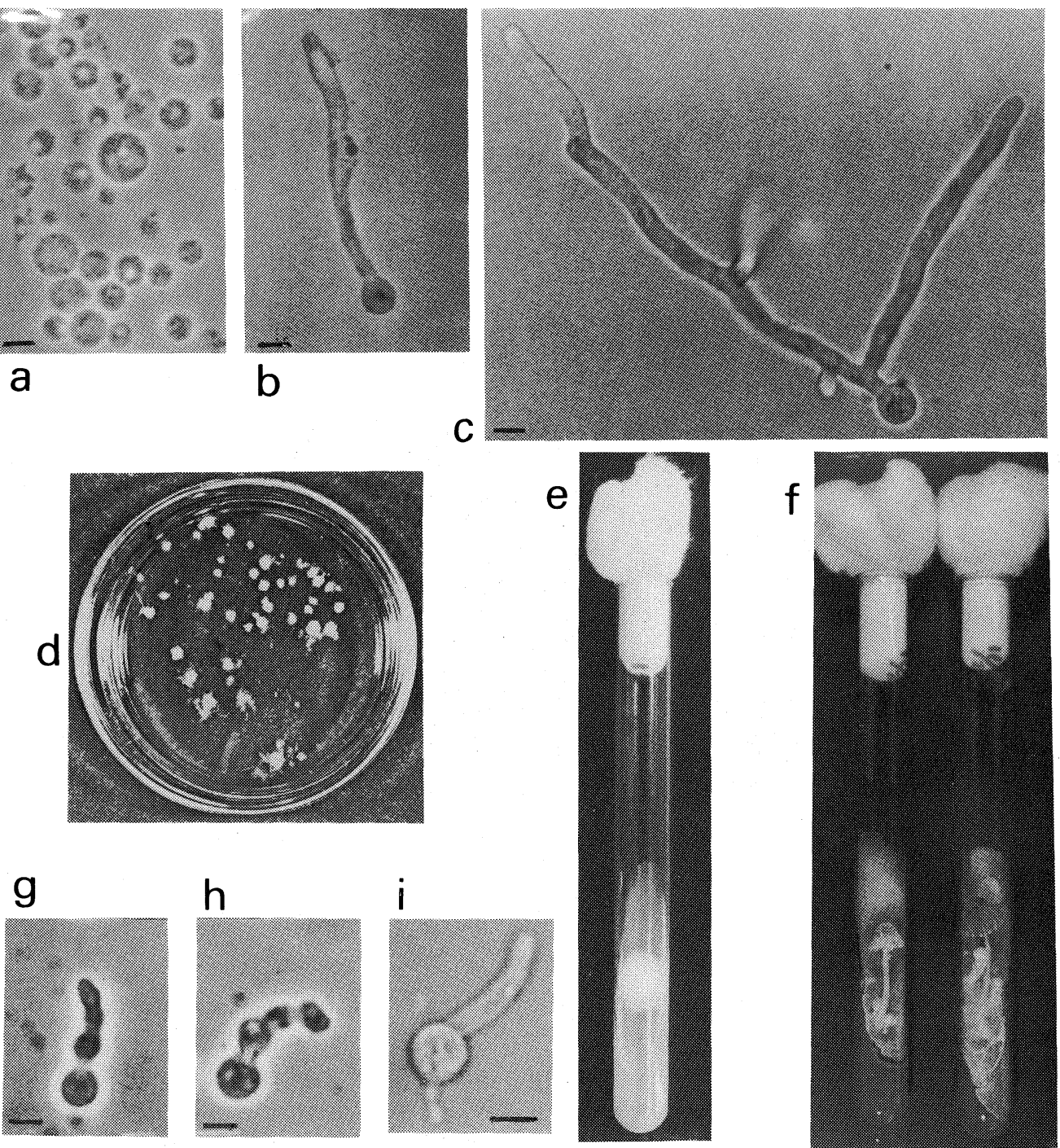

Fig. 2. Mycelial Protoplasts of Coprinus macrorhizus Fis $^{\mathrm{c}}$ and Their Development.

a: Freshly isolated protoplasts. The bar represents $5 \mu$.

b: An early stage of hyphal growth from a protoplast on an agar plate.

c: A later stage of hyphal growth from from a protoplast on an agar plate.

$\mathrm{d}$ : Colonies derived from protoplasts on an agar plate.

e: Growth of a colony transferred to an agar slant containing no osmotic stabilizer.

$f$ : Formation of fruiting bodies by mycelia regenerated from protoplasts.

$\mathrm{g} \sim \mathrm{i}$ : Abnormal development of hyphae from protoplasts in a liquid medium.

taminated the protoplast suspension. The numbers of colonies formed on the plates without osmoticum were subtracted from those on the plates containing an osmoticum, when the plating efficiency of protoplasts was to be precisely determined.
Some factors influencing the plating efficiency of protoplasts were studied. As shown in Fig. 3a, sucrose, $\mathrm{MgSO}_{4}$, mannitol, sorbitol and glucose added at $0.5 \mathrm{M}$ to the regeneration medium (MYG) were all effective as osmotic stabilizers for colony formation, but 


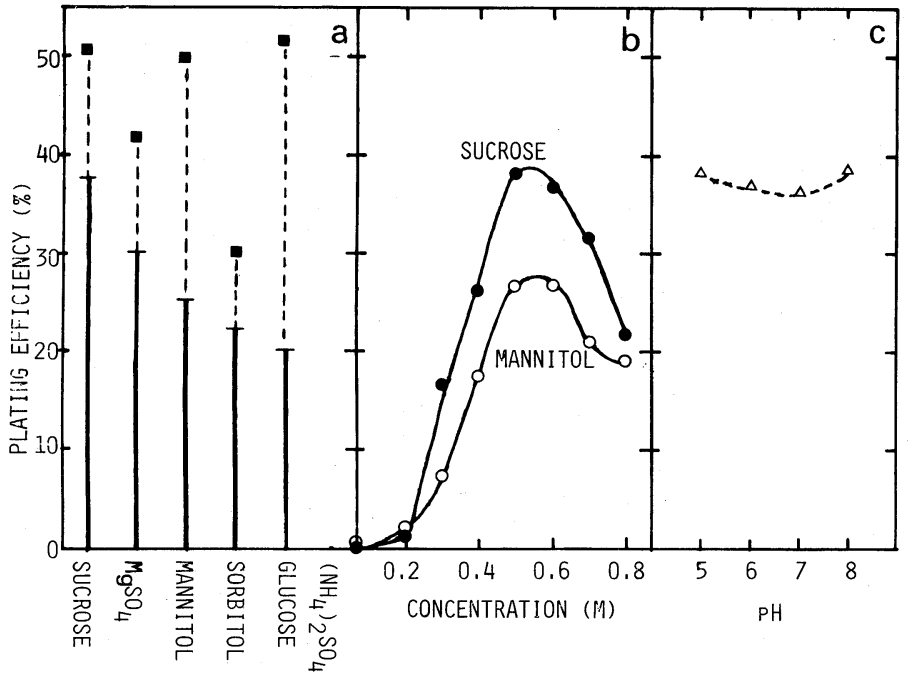

FIG. 3. Effects of Osmotic Stabilizers and pH of the Medium on Plating Efficiency of Coprinus macrorhizus Fis ${ }^{c}$ Protoplasts.

Protoplasts were isolated using the standard enzyme solution.

a : Effects of Various Osmotic Stabilizers. Protoplasts were plated on agar $(1.2 \%)$ plates of MYG medium containing the indicated osmotic stabilizers at $0.5 \mathrm{M}$. Solid lines represent the average plating efficiency for $2 \sim 10$ experiments. The symbol ( $\mathbf{a})$ with a dotted line denotes the highest plating efficiency obtained.

b : Effects of Concentrations of Osmotic Stabilizers. Protoplasts were plated on agar (1.2\%) plates of MYG medium containing sucrose $(\bigcirc)$ or mannitol $(\bigcirc)$ at the indicated concentrations.

$\mathrm{c}$ : Effects of $\mathrm{pH}$. Protoplasts were plated on agar $(1.2 \%)$ plates of MYG medium containing $0.5 \mathrm{~m}$ sucrose. The $\mathrm{pH}$ of the medium had been adjusted to the indicated values.

$\left(\mathrm{NH}_{4}\right)_{2} \mathrm{SO}_{4}$ was not. Sucrose gave the best results both in terms of average and maximal efficiency (37 and $51 \%$, respectively). With other osmotica the plating efficiency fluctuated considerably from experiment to experiment, and the growth of colonies was sometimes very slow. The osmoticum concentration optimal for colony formation was $0.5 \sim 0.6 \mathrm{M}$ with mannitol and sucrose (Fig. 3b). The plating efficiency was little affected by the $\mathrm{pH}$ of the culture medium in the range of 5 to 8 (Fig. 3c).

The plating efficiency of $C$. macrorhizus protoplasts was also influenced by the enzymes used for their isolation (Table II). The inclusion of Novozyme 234 but not Zymolyase in the enzyme solution caused a reduction in the efficiency of colony formation. This was paralleled by a reduction in the proportion of nucleated protoplasts (Table II). The protoplasts isolated early in the enzyme reaction tended to show a lower efficiency of colony formation.
The plating efficiency of protoplasts varied with the strains of $C$. macrorhizus and was influenced by the composition of the medium (Table III). Although protoplasts could be obtained from the four strains in similar yields, their plating efficiencies varied widely. The wild type and mutant $\mathrm{Fis}^{\mathrm{c}}$ formed colonies with efficiencies of over $30 \%$ on MYG medium, and with efficiencies of nearly $20 \%$ even on the minimal medium. In contrast, auxotrophic mutants 5007 and 5132 formed few, if any, colonies even on MYG medium. The plating efficiency of strain 5010 could be improved significantly by adding peptone and $\mathrm{N}$-acetylglucosamine to the MYG medium. Non-acetylated glucosamine had no effect. $N$-acetyl glucosamine also improved the plating efficiency of $\mathrm{Fis}^{\mathfrak{c}}$ protoplasts both in MYG and minimal medium.

When the colonies formed by $\mathrm{Fis}^{\mathrm{c}}$ protoplasts were transferred to agar MYG medium containing no osmoticum, they grew 
Table III. Effects of the Medium Condition on Plating Efficiency of Coprinus macrorhizus Protoplasts

\begin{tabular}{|c|c|c|c|c|}
\hline \multirow{2}{*}{ Medium $^{a}$} & \multicolumn{4}{|c|}{ Strains } \\
\hline & Wild type & $\mathrm{Fis}^{\mathrm{c}}$ & $5010^{b}$ & $5132^{c}$ \\
\hline MYG & 31 & 35 & 0.5 & 0 \\
\hline MYG + peptone ${ }^{d}(0.4 \%)$ & 30 & 36 & 11 & 0.01 \\
\hline MYG + glucosamine $(0.4 \%)$ & & 30 & & 0 \\
\hline MYG $+N$-acetyl glucosamine $(0.4 \%)$ & & 41 & 5 & 0 \\
\hline MIN (minimal medium) & 17 & 19 & 0 & 0 \\
\hline MIN + glucosamine $(0.4 \%)$ & & 13 & 0 & 0 \\
\hline MIN $+N$-acetyl glucosamine $(0.4 \%)$ & & 29 & 0 & 0 \\
\hline
\end{tabular}

rapidly (Fig. 2e) and developed fruiting bodies within $5 \sim 7$ days (Fig. 2f). the fruiting bodies produced normal spores. Remarkably, the colonies derived from protoplasts formed fruiting bodies without exception whereas those derived from conventional inocula (spores, odia and mycelial fragments) occasionally failed to do so.

\section{DISCUSSION}

The mycelial walls of $C$. macrorhizus were effectively digested with a mixture of Streptomyces griseus chitinase, Tricoderma viride cellulase and Arthrobacter luteus glucanase, all available commercially. Over $2 \times 10^{8}$ protoplasts per $\mathrm{ml}$ of enzyme solution were consistently obtained with the mixture within $2 \mathrm{hr}$ incubation. The yield is higher by at least one order of magnitude than the values so far reported for basidiomycetes.

It is reasonable to assume that chitinase (poly $(1,4-\beta$-(2-acetamido-2-deoxy-D-glucosamine))-glycano-hydrolase) plays a critical role in the release of $C$. macrorhizus protoplasts. Chitin is a major cell wall component of basidiomycetes, and DeVries and Wessels showed using a purified preparation that chitinase is essential for the release of protoplasts from $S$. commune mycelia. ${ }^{4)}$ On the other hand, it is not clear whether cellulase
(1,4-(1,3; 1,4)- $\beta$-D-glucan-4-glucanohydrolase) is involved in the release of $C$. macrorhizus protoplasts. DeVries and Wessels showed that a culture filtrate of Trichoderma viride, which is devoid of cellulolytic activity, digested the mycelial walls of $S$. commune. ${ }^{3)}$ These workers showed also that, in addition to chitinase, $\alpha$ glucanase is essential for the release of protoplasts from $S$. commune mycelia. ${ }^{4}$ It is not known if this enzyme is present in the Cellulase Onozuka used in our study, whereas the preparation is known to contain $\beta$-1,3-glucanase. Possible involvement of the latter enzyme in the release of $C$. macrorhizus protoplasts is suggested by the observation that Zymolyase, a $\beta$-1,3-glucanase active on yeast cell walls, ${ }^{17}$ ) enhanced protoplast release when added to chitinase (Table I).

Our method developed for isolating $C$. macrorhizus protoplasts is characterized not only by the high yield but also by the excellent viability of the isolated protoplasts. This is indicated by the fact that a large proportion of $C$. macrorhizus wild type and Fis ${ }^{\mathrm{c}}$ protoplasts were capable of regenerating hyphae and growing into colonies. The plating efficiency of $50 \%$ attained in some experiments is probably the upper limit, because the proportion of nucleated protoplasts did not exceed $50 \%$. Regeneration of hyphae via aberrant structures is common with protoplasts 
of filamentous fungi and basidiomycetes, ${ }^{4,11)}$ but was very rarely seen in our C. macrorhizus protoplasts.

It is noteworthy that a powerful enzyme does not necessarily yield highly viable protoplasts. Novozyme 234 was very powerful for digesting C. macrorhizus mycelia, but the protoplasts isolated using this enzyme were smaller in size, contained a larger proportion of enucleated protoplasts and showed lower plating efficiency as compared with the protoplasts isolated using the standard enzyme mixture.

We found that $N$-acetyl glucosamine, the repeating unit of chitin, enhanced colony formation by $C$. macrorhizus protoplasts, when added to the complex MYG medium and to the synthetic minimal medium (Table III). This finding suggests that the plating efficiency in the minimal medium may be improved further by creating conditions favorable for cell wall biosynthesis. For unknown reasons, non acetylated glucosamine was not effective in this respect.

Colonies derived from $C$. macrorhizus $\mathrm{Fis}^{\mathrm{c}}$ protoplasts consistently formed fruiting bodies when transferred onto MYG medium containing no osmoticum. Although direct regeneration of fruiting bodies without prior mating may be a unique property of the protoplasts of Fis $^{c}$, a mutant capable of forming monokaryotic fruiting bodies, this observation indicates the possibility of fruiting body formation from the fusion products between protoplasts of different mating types. Our recent experiments using two auxotrophic mutants of C. macrorhizus show that this is in fact the case (in preparation).
Acknowledgments. The authors are grateful to Dr. T. Takemaru and Dr. T. Kamada for valuable discussions and for the mutant strains of $C$. macrorhizus. Thanks are also due to Dr. T. Ishikawa for providing the Fis ${ }^{\mathfrak{c}}$ strain of C. macrorhizus. Novozyme 234 was kindly given by Dr. T. Nagata, National Institute for Basic Biology. This work was supported by a project grant from the Ministry of Agriculture, Forestry and Fisheries of Japan.

\section{REFERENCES}

1) L. Ferenczy, "Genetics as a Tool in Microbiology," ed. by S. W. Glovar and D. A. Hopwood, Cambridge Univ. Press, Cambridge, 1981, p. 1.

2) D. A. Hopwood, Ann. Rev. Microbiol., 35, 237 (1981).

3) O. M. H. DeVries and J. G. H. Wessels, J. Gen. Microbiol., 73, 13 (1972).

4) O. M. H. DeVries and J. G. H. Wessels, Arch. Microbiol., 102, 209 (1975).

5) O. M. H. DeVries and J. G. H. Wessels, J. Gen. Microbiol., 76, 319 (1973).

6) D. Moore, M. M. Y. Elhiti and R. D. Butler, New Phytologist, 83, 695 (1979).

7) D. Moore, Trans. British Micol. Soc., 65, 134 (1975).

8) K. Akamatsu, T. Kamada and T. Takemaru, Trans. Mycol. Soc. Jpn., 24, 173 (1983).

9) R. Ushiyama and Y. Nakai, Rept. Tottori Mycol. Inst., 15, 1 (1977).

10) M. Abe, H. Umetsu, T. Nakai and D. Sasage, Agric. Biol. Chem., 46, 1955 (1982).

11) O. Yamada, Y. Magae, Y. Kashiwagi, Y. Kakimoto and T. Sasaki, Appl. Microbiol. Biotech., 17, 298 (1983).

12) S. O. Yanagi and I. Takebe, Appl. Microbiol. Biotech., 19, 58 (1984).

13) I. Uno and T. Ishikawa, Molec. Gen. Genetics, 113, 228 (1971).

14) H. Maeda and N. Ishida, J. Biochem., 62, 276 (1967).

15) T. Nagata and I. Takebe, Planta, 92, 301 (1970).

16) T. Kuroiwa and T. Suzuki, Cell Structure and Function, 5, 195 (1980).

17) K. Kitamura and Y. Yamamoto, Arch. Biochem. Biophys., 153, 403 (1972). 